To appear in American Mathematical Monthly

\title{
An identity involving the least common multiple of binomial coefficients and its application
}

\author{
BAKIR FARHI \\ bakir.farhi@gmail.com
}

\begin{abstract}
In this paper, we prove the identity

$$
\operatorname{lcm}\left\{\left(\begin{array}{l}
k \\
0
\end{array}\right),\left(\begin{array}{l}
k \\
1
\end{array}\right), \ldots,\left(\begin{array}{l}
k \\
k
\end{array}\right)\right\}=\frac{\operatorname{lcm}(1,2, \ldots, k, k+1)}{k+1} \quad(\forall k \in \mathbb{N}) .
$$

As an application, we give an easily proof of the well-known nontrivial lower bound $\operatorname{lcm}(1,2, \ldots, k) \geq 2^{k-1}(\forall k \geq 1)$.
\end{abstract}

MSC: $11 \mathrm{~A} 05$.

Keywords: Least common multiple; Binomial coefficients; Kummer's theorem.

\section{Introduction and Results}

Many results concerning the least common multiple of a sequence of integers are known. The most famous is nothing else than an equivalent of the prime number theorem; it states that $\log \operatorname{lcm}(1,2, \ldots, n) \sim n$ as $n$ tends to infinity (see, e.g., [4]). Effective bounds for $\operatorname{lcm}(1,2, \ldots, n)$ are also given by several authors. Among others, Nair [7] discovered a nice new proof for the wellknown estimate $\operatorname{lcm}(1,2, \ldots, n) \geq 2^{n-1}(\forall n \geq 1)$. Actually, Nair's method simply exploits the integral $\int_{0}^{1} x^{n}(1-x)^{n} d x$. Further, Hanson [3] already obtained the upper bound $\operatorname{lcm}(1,2, \ldots, n) \leq 3^{n}(\forall n \geq 1)$.

Recently, many related questions and many generalizations of the above results have been studied by several authors. The interested reader is referred to [1], [2], and [5].

In this note, using Kummer's theorem on the $p$-adic valuation of binomial coefficients (see, e.g., [6]), we obtain an explicit formula for $\operatorname{lcm}\left\{\left(\begin{array}{l}k \\ 0\end{array}\right),\left(\begin{array}{l}k \\ 1\end{array}\right), \ldots,\left(\begin{array}{l}k \\ k\end{array}\right)\right\}$ 
in terms of the least common multiple of the first $k+1$ consecutive positive integers. Then, we show how the well-known nontrivial lower bound $\operatorname{lcm}(1,2, \ldots, n) \geq 2^{n-1}(\forall n \geq 1)$ can be deduced very easily from that formula. Our main result is the following:

Theorem 1 For any $k \in \mathbb{N}$, we have:

$$
\operatorname{lcm}\left\{\left(\begin{array}{l}
k \\
0
\end{array}\right),\left(\begin{array}{l}
k \\
1
\end{array}\right), \ldots,\left(\begin{array}{l}
k \\
k
\end{array}\right)\right\}=\frac{\operatorname{lcm}(1,2, \ldots, k, k+1)}{k+1} .
$$

First, let us recall the so-called Kummer's theorem:

Theorem (Kummer [6]) Let $n$ and $k$ be natural numbers such that $n \geq k$ and let $p$ be a prime number. Then the largest power of $p$ dividing $\left(\begin{array}{l}n \\ k\end{array}\right)$ is given by the number of borrows required when subtracting $k$ from $n$ in the base $p$.

Note that the last part of the theorem is also equivalently stated as the number of carries when adding $k$ and $n-k$ in the base $p$.

As usually, if $p$ is a prime number and $\ell \geq 1$ is an integer, we let $v_{p}(\ell)$ denote the normalized $p$-adic valuation of $\ell$; that is, the exponent of the largest power of $p$ dividing $\ell$. We first prove the following proposition.

Proposition 2 Let $k$ be a natural number and $p$ a prime number. Let $k=$ $\sum_{i=0}^{N} c_{i} p^{i}$ be the $p$-base expansion of $k$, where $N \in \mathbb{N}, c_{i} \in\{0,1, \ldots, p-1\}$ (for $i=0,1, \ldots, N)$ and $c_{N} \neq 0$. Then we have:

$\max _{0 \leq \ell \leq k} v_{p}\left(\left(\begin{array}{l}k \\ \ell\end{array}\right)\right)=v_{p}\left(\left(\begin{array}{c}k \\ p^{N}-1\end{array}\right)\right)= \begin{cases}0 & \text { if } k=p^{N+1}-1 \\ N-\min \left\{i \mid c_{i} \neq p-1\right\} & \text { otherwise. }\end{cases}$

Proof. We distinguish the following two cases:

$\mathbf{1}^{\text {st }}$ case. If $k=p^{N+1}-1$ :

In this case, we have $c_{i}=p-1$ for all $i \in\{0,1, \ldots, N\}$. So it is clear that in base $p$, the subtraction of any $\ell \in\{0,1, \ldots, k\}$ from $k$ doesn't require any borrows. It follows from Kummer's theorem that $v_{p}\left(\left(\begin{array}{l}k \\ \ell\end{array}\right)\right)=0, \forall \ell \in$ $\{0,1, \ldots, k\}$. Hence

$$
\max _{0 \leq \ell \leq k} v_{p}\left(\left(\begin{array}{l}
k \\
\ell
\end{array}\right)\right)=v_{p}\left(\left(\begin{array}{c}
k \\
p^{N}-1
\end{array}\right)\right)=0
$$

as required. 
$2^{\text {nd }}$ case. If $k \neq p^{N+1}-1$ :

In this case, at least one of the digits of $k$, in base $p$, is different from $p-1$. So we can define:

$$
i_{0}:=\min \left\{i \mid c_{i} \neq p-1\right\} .
$$

We have to show that for any $\ell \in\{0,1, \ldots, k\}$, we have $v_{p}\left(\left(\begin{array}{l}k \\ \ell\end{array}\right)\right) \leq N-i_{0}$, and that $v_{p}\left(\left(\begin{array}{c}k \\ p^{N}-1\end{array}\right)\right)=N-i_{0}$.

Let $\ell \in\{0,1, \ldots, k\}$ be arbitrary. Since (by the definition of $\left.i_{0}\right) c_{0}=c_{1}=$ $\cdots=c_{i_{0}-1}=p-1$, during the process of subtraction of $\ell$ from $k$ in base $p$, the first $i_{0}$ subtractions digit-by-digit don't require any borrows. So the number of borrows required in the subtraction of $\ell$ from $k$ in base $p$ is at most equal to $N-i_{0}$. According to Kummer's theorem, this implies that $v_{p}\left(\left(\begin{array}{c}k \\ \ell\end{array}\right)\right) \leq N-i_{0}$.

Now, consider the special case $\ell=p^{N}-1=\sum_{i=0}^{N-1}(p-1) p^{i}$. Since $c_{0}=c_{1}=$ $\cdots=c_{i_{0}-1}=p-1$ and $c_{i_{0}}<p-1$, during the process of subtraction of $\ell$ from $k$ in base $p$, each of the subtractions digit-by-digit from the rank $i_{0}$ to the rank $N-1$ requires a borrow. It follows from Kummer's theorem that $v_{p}\left(\left(\begin{array}{c}k \\ p^{N}-1\end{array}\right)\right)=N-i_{0}$. This completes the proof of the proposition.

Now we are ready to prove our main result.

Proof of Theorem 1. The identity of Theorem 1 is satisfied for $k=0$. For the following, suppose $k \geq 1$. Equivalently, we have to show that

$$
v_{p}\left(\operatorname{lcm}\left\{\left(\begin{array}{l}
k \\
0
\end{array}\right),\left(\begin{array}{l}
k \\
1
\end{array}\right), \ldots,\left(\begin{array}{l}
k \\
k
\end{array}\right)\right\}\right)=v_{p}\left(\frac{\operatorname{lcm}(1,2, \ldots, k, k+1)}{k+1}\right),
$$

for any prime number $p$.

Let $p$ be an arbitrary prime number and $k=\sum_{i=0}^{N} c_{i} p^{i}$ be the $p$-base expansion of $k$ (where $N \in \mathbb{N}, c_{i} \in\{0,1, \ldots, p-1\}$ for $i=0,1, \ldots, N$, and $\left.c_{N} \neq 0\right)$. By Proposition 2, we have

$$
\begin{aligned}
v_{p}\left(\operatorname{lcm}\left\{\left(\begin{array}{l}
k \\
0
\end{array}\right),\left(\begin{array}{l}
k \\
1
\end{array}\right), \ldots,\left(\begin{array}{l}
k \\
k
\end{array}\right)\right\}\right)=\max _{0 \leq \ell \leq k} v_{p}\left(\left(\begin{array}{l}
k \\
\ell
\end{array}\right)\right) \\
= \begin{cases}0 & \text { if } k=p^{N+1}-1 \\
N-\min \left\{i \mid c_{i} \neq p-1\right\} & \text { otherwise. }\end{cases}
\end{aligned}
$$

Next, it is clear that $v_{p}(\operatorname{lcm}(1,2, \ldots, k, k+1))$ is equal to the exponent of the largest power of $p$ not exceeding $k+1$. Since (according to the expansion of $k$ in base $p$ ) the largest power of $p$ not exceeding $k$ is $p^{N}$, the largest power of $p$ not exceeding $k+1$ is equal to $p^{N+1}$ if $k+1=p^{N+1}$ and equal to $p^{N}$ if $k+1 \neq p^{N+1}$. Hence, we have

$$
v_{p}(\operatorname{lcm}(1,2, \ldots, k, k+1))= \begin{cases}N+1 & \text { if } k=p^{N+1}-1 \\ N & \text { otherwise }\end{cases}
$$


Further, it is easy to verify that

$$
v_{p}(k+1)= \begin{cases}N+1 & \text { if } k=p^{N+1}-1 \\ \min \left\{i \mid c_{i} \neq p-1\right\} & \text { otherwise. }\end{cases}
$$

By subtracting the relation (44) from the relation (3) and using an elementary property of the $p$-adic valuation, we obtain

$$
v_{p}\left(\frac{\operatorname{lcm}(1,2, \ldots, k, k+1)}{k+1}\right)= \begin{cases}0 & \text { if } k=p^{N+1}-1 \\ N-\min \left\{i \mid c_{i} \neq p-1\right\} & \text { otherwise. }\end{cases}
$$

The required equality (1) follows by comparing the two relations (2) and (5).

\section{Application to prove a nontrivial lower bound for $\operatorname{lcm}(1,2, \ldots, n)$}

We now apply Theorem 1 to obtain a nontrivial lower bound for the numbers $\operatorname{lcm}(1,2, \ldots, n)(n \geq 1)$.

Corollary 3 For all integer $n \geq 1$, we have:

$$
\operatorname{lcm}(1,2, \ldots, n) \geq 2^{n-1} .
$$

Proof. Let $n \geq 1$ be an integer. By applying Theorem 1 for $k=n-1$, we have:

$$
\begin{aligned}
\operatorname{lcm}(1,2, \ldots, n) & =n \cdot \operatorname{lcm}\left\{\left(\begin{array}{c}
n-1 \\
0
\end{array}\right),\left(\begin{array}{c}
n-1 \\
1
\end{array}\right), \ldots,\left(\begin{array}{c}
n-1 \\
n-1
\end{array}\right)\right\} \\
& \geq n \cdot \max _{0 \leq i \leq n-1}\left(\begin{array}{c}
n-1 \\
i
\end{array}\right) \\
& \geq \sum_{i=0}^{n-1}\left(\begin{array}{c}
n-1 \\
i
\end{array}\right)=2^{n-1},
\end{aligned}
$$

as required. The corollary is proved.

\section{References}

[1] P. Bateman, J. Kalb, and A. Stenger, A limit involving least common multiples, Amer. Math. Monthly. 109 (2002) 393-394. 
[2] B. Farhi, Nontrivial lower bounds for the least common multiple of some finite sequences of integers, J. Number Theory. 125 (2007) 393-411.

[3] D. Hanson, On the product of the primes, Canad. Math. Bull. 15 (1972) 33-37.

[4] G. H. Hardy and E. M. Wright, The Theory of Numbers, 5th ed., Oxford University. Press, London, 1979.

[5] S. Hong and W. Feng, Lower bounds for the least common multiple of finite arithmetic progressions, C. R. Math. Acad. Sci. Paris. 343 (2006) 695-698.

[6] E. E. Kummer, Über die Ergänzungssätze zu den allgemeinen Reciprocitätsgesetzen, J. Reine Angew. Math. 44 (1852) 93-146.

[7] M. Nair, On Chebyshev-type inequalities for primes, Amer. Math. Monthly. 89 (1982) 126-129. 